\title{
Numerical Analysis of the Fractional-Order Nonlinear System of Volterra Integro-Differential Equations
}

\author{
Pongsakorn Sunthrayuth $\mathbb{D}^{1},{ }^{1}$ Roman Ullah, ${ }^{2}$ Adnan Khan, ${ }^{3}$ Rasool Shah $\mathbb{D}^{3}{ }^{3}$ \\ Jeevan Kafle $\mathbb{D}^{4},{ }^{4}$ Ibrahim Mahariq ${ }^{(D},{ }^{5}$ and Fahd Jarad ${ }^{6}{ }^{6,7}$ \\ ${ }^{1}$ Department of Mathematics and Computer Science, Faculty of Science and Technology, Rajamangala University of Technology \\ Thanyaburi (RMUTT), Thanyaburi, Pathum Thani, Thailand \\ ${ }^{2}$ Department of Computing, Muscat College, Muscat, Oman \\ ${ }^{3}$ Department of Mathematics, Abdul Wali Khan University Mardan, Mardan 23200, Pakistan \\ ${ }^{4}$ Central Department of Mathematics, Tribhuvan University, Kirtipur, Kathmandu, Nepal \\ ${ }^{5}$ College of Engineering and Technology, American University of the Middle East, Kuwait \\ ${ }^{6}$ Department of Mathematics, Cankaya University, Etimesgut, Ankara, Turkey \\ ${ }^{7}$ Department of Medical Research, China Medical University Hospital, China Medical University, Taichung, Taiwan
}

Correspondence should be addressed to Jeevan Kafle; jeevan.kafle@cdmath.tu.edu.np and Fahd Jarad; fahd@cankaya.edu.tr

Received 15 July 2021; Revised 30 August 2021; Accepted 8 October 2021; Published 21 October 2021

Academic Editor: Emanuel Guariglia

Copyright (C) 2021 Pongsakorn Sunthrayuth et al. This is an open access article distributed under the Creative Commons Attribution License, which permits unrestricted use, distribution, and reproduction in any medium, provided the original work is properly cited.

\begin{abstract}
This paper presents the nonlinear systems of Volterra-type fractional integro-differential equation solutions through a Chebyshev pseudospectral method. The proposed method is based on the Caputo fractional derivative. The results that we get show the accuracy and reliability of the present method. Different nonlinear systems have been solved; the solutions that we get are compared with other methods and the exact solution. Also, from the presented figures, it is easy to conclude that the CPM error converges quickly as compared to other methods. Comparing the exact solution and other techniques reveals that the Chebyshev pseudospectral method has a higher degree of accuracy and converges quickly towards the exact solution. Moreover, it is easy to implement the suggested method for solving fractional-order linear and nonlinear physical problems related to science and engineering.
\end{abstract}

\section{Introduction}

Fractional calculus has a long history as classical calculus. The concept of fractional calculus arouse when Leibnitz used a proper representation $d^{n} f / d x^{n}$ for the $n$th derivative in his publications. L'hopital raises a question on the particular notation on what happens if " $n$ " is a noninteger. It was the beginning of fractional calculus [1]. Recently, mathematicians focused on fractional calculus due to its numerous applications in every field of science: viscoelastic materials [2], economics [3], continuum and statistical mechanics [4], dynamics of interfaces between soft nanoparticles and rough substrates [5], solid mechanics [6], and much more [7-14].
Mathematical formulations solve many problems of nature with the help of converting the physical phenomena to the equation form. Differential equations (DEs) are among those that play the main role in modeling various phenomena. However, some problems are complex and cannot be handled with the help of a differential equation. In this regard, the researchers utilized fractional differential equations (FDEs) that model the phenomenon more accurately than differential equations having order integers. Nowadays, FDEs got the importance of real-world modeling problems: such as electrode-electrolyte polarization [15], electrochemistry of corrosion [16], circuit systems [17], optics and signal processing [18], heat conduction [19], diffusion wave [20], control theory of dynamical systems [21], 
fluid flow [22], probability and statistics [23, 24], and so on (see [25-28]).

The role of fractional integral and integrodifferential equations is found in every field of engineering and science. When a physical phenomenon is modeled under the differential equation, it finally gives a differential equation, an integral equation, or an integrodifferential equation. Some applications of these types of equations are nanohydrodynamics [29], glass-forming process [30], wind ripple in the desert [31], and drop-wise condensation [32]. The analytical solution of integral and integrodifferential equations does not exist in most cases. Even if it exists in certain cases, it is hard to find. Different numerical methods have been developed for finding an approximate solution of integral and integro-differential equations. The most common among these methods are the Chebyshev polynomials [33], Haar wavelet [34], triangular function method [35], collocation method [36], Legendre wavelet operational method [37], Taylor series expansion method [38], homotopy perturbation method [39], reproducing kernel Hilbert space method [40], Adomian decomposition method [41], Euler wavelet method [42], variational iteration method [43], spectral collocation method [44], least square method [45], homotopy analysis method [46], and differential transform method [47].

We apply Chebyshev pseudospectral method (CPM) to solve nonlinear Volterra integro-differential equation systems in the present work. CPM is a powerful technique for solving linear and nonlinear problems. The obtained results show the higher convergence rate of the present technique. The solution that we get shows that CPM has good agreement with the exact solution. Error analysis reveals the efficiency of the proposed technique that CPM has greater accuracy than other methods.

\section{Definitions and Preliminary Concept}

This unit shows the preliminary concept and some essential definitions taken from fractional calculus and used in our present research work.

2.1. Definition. The definition for fractional derivative by Caputo of order $\alpha$ is showed by the following mathematical expression [48]:

$$
D^{\alpha} j(s)=\frac{1}{\Gamma(n-\alpha)} \int_{0}^{s}(s-t)^{n-\alpha-1} j^{(n)}(t) d t,
$$

for $n-1<\alpha \leq n, n \in \mathbb{N}, s>0, j \in \mathbb{C}_{-1}^{m}$.

2.2. Definition. The fractional derivatives by Jin-Hunan He are described as [48]

$$
\frac{D^{\alpha} j(s)}{D s^{\alpha}}=\Gamma(1+\alpha) \lim _{\Delta s=s_{1}-s_{2}} \longrightarrow L \frac{f\left(s_{1}\right)-f\left(s_{2}\right)}{\left(s_{1}-s_{2}\right)^{\alpha}},
$$

where $\Delta s$ does not approach zero.
2.3. Definition. Xiao-Jun explains derivatives having fractional order as [48]

$$
D_{s}^{\alpha} j\left(s_{0}\right)=j^{\alpha}\left(s_{0}\right)=\left.\frac{d^{\alpha} j(s)}{d s^{\alpha}}\right|_{s=s_{0}}=\lim _{s \longrightarrow s_{0}} \frac{\Delta^{\alpha}\left(j(s)-j\left(s_{0}\right)\right)}{\left(s-s_{0}\right)^{\alpha}},
$$

where

$$
\Delta^{\alpha}\left(j(s)-j\left(s_{0}\right)\right) \cong \Gamma(1+\alpha) \Delta\left(j(s)-j\left(s_{0}\right)\right)
$$

2.4. Definition. The integral operator by Riemann-Liouville for order $\alpha$ is [48]

$$
I^{\alpha} j(s)=\frac{1}{\Gamma(\alpha)} \int_{0}^{s}(s-t)^{\alpha-1} j(t) d t
$$

The Caputo derivative operator and Riemann-Liouville integral operator have the following properties

$$
\begin{aligned}
& D^{\alpha} I^{\alpha} j(s)=j(s), \\
& I^{\alpha} D^{\alpha} j(s)=j(s)-\sum_{k=0}^{n-1} \frac{j^{(k)}\left(0^{+}\right)}{k !} s^{k}, s \geq 0 n-1<\alpha<n .
\end{aligned}
$$

\section{Chebyshev Pseudospectral Method (CPM)}

The Chebyshev polynomials are defined in the $[-1,1]$ interval and can be described by the following recurrence formula:

$$
R_{n+1}(t)=2 u R_{n}(s)-R_{n-1}(s), \quad n=1,2, \cdots,
$$

where

$$
\begin{aligned}
& R_{0}(s)=1, \\
& R_{1}(s)=s .
\end{aligned}
$$

To apply the Chebyshev polynomials in the $[0,1]$ interval, we define the Chebyshev shifted polynomials $\widehat{R}_{n}(s)$ which are defined in the manner of Chebyshev polynomials $R_{n}(s)$ by relation

$$
\widehat{R}_{n}(s)=R_{n}(2 s-1)
$$

And the recurrence formula is as follows:

$$
\widehat{R}_{n+1}(s)=2(2 s-1) \widehat{R}_{n}(s)-\widehat{R}_{n-1}(s), \quad n=1,2, \cdots,
$$

where

$$
\begin{aligned}
& \widehat{R}_{0}(s)=1, \\
& \widehat{R}_{1}(s)=2 s-1 .
\end{aligned}
$$


TABLE 1: Exact versus CPM solution of problem 1 at $m=10$.

\begin{tabular}{lcccc}
\hline$s$ & Exact $j(s)$ & Exact $k(s)$ & CPM solution $j(s)$ & CPM solution $k(s)$ \\
\hline 0 & 0.0000000000 & 1.0000000000 & 0.0000000000 & 1.0000000000 \\
0.1 & 0.1001667500 & 1.0050041680 & 0.1001667500 & 1.0050041680 \\
0.2 & 0.2013360025 & 1.0200667556 & 0.2013360025 & 1.0200667556 \\
0.3 & 0.3045202934 & 1.0453385141 & 0.3045202934 & 1.0453385141 \\
0.4 & 0.4107523258 & 1.0810723718 & 0.4107523258 & 1.0810723718 \\
0.5 & 0.5210953054 & 1.1276259652 & 0.5210953054 & 1.1276259652 \\
0.6 & 0.6366535821 & 1.1854652182 & 0.6366535821 & 1.1854652182 \\
0.7 & 0.7585837018 & 1.2551690056 & 0.7585837018 & 1.2551690056 \\
0.8 & 0.8881059821 & 1.3374349463 & 0.8881059821 & 1.3374349462 \\
0.9 & 1.0265167257 & 1.4330863854 & 1.0265167253 & 1.4330863853 \\
1.0 & 1.1752011936 & 1.5430806348 & 1.1752011918 & 1.5430806343 \\
\hline
\end{tabular}

TABLE 2: Error comparison of CPM versus other methods of Section 4.1 at $m=10$.

\begin{tabular}{lcccc}
\hline$s$ & Error $\left(j_{\mathrm{CPM}}\right)$ & Error $\left(k_{\mathrm{CPM}}\right)$ & Error $\left(j_{\mathrm{OTM}}\right)$ & Error $\left(k_{\mathrm{OTM}}\right)$ \\
\hline 0 & $0.0000000000 E+00$ & $0.0000000000 E+00$ & $0.00 E+00$ & $0.00 E+00$ \\
0.1 & $8.2692537238 E-17$ & $1.4541999963 E-17$ & $1.39 E-17$ & $0.00 E+00$ \\
0.2 & $9.1822482293 E-16$ & $1.6662883612 E-16$ & $5.27 E-16$ & $0.00 E+00$ \\
0.3 & $1.2443994903 E-15$ & $2.2699378388 E-16$ & $4.45 E-14$ & $1.11 E-15$ \\
0.4 & $1.2607906760 E-15$ & $2.3113111594 E-16$ & $1.05 E-12$ & $3.51 E-14$ \\
0.5 & $7.2897283903 E-16$ & $1.2454060032 E-16$ & $1.23 E-11$ & $5.10 E-13$ \\
0.6 & $2.5175064758 E-13$ & $5.5241090380 E-14$ & $9.11 E-11$ & $4.55 E-12$ \\
0.7 & $5.8189005235 E-12$ & $1.3209327312 E-12$ & $4.97 E-10$ & $2.16 E-9$ \\
0.8 & $5.8725732247 E-11$ & $1.3786485036 E-11$ & $7.90 E-9$ & $2.90 E-11$ \\
0.9 & $3.7796170567 E-10$ & $9.1669608328 E-11$ & $2.52 E-8$ & $2.10 E-9$ \\
1.0 & $1.8157658168 E-09$ & $4.5450903393 E-10$ & & \\
\hline
\end{tabular}

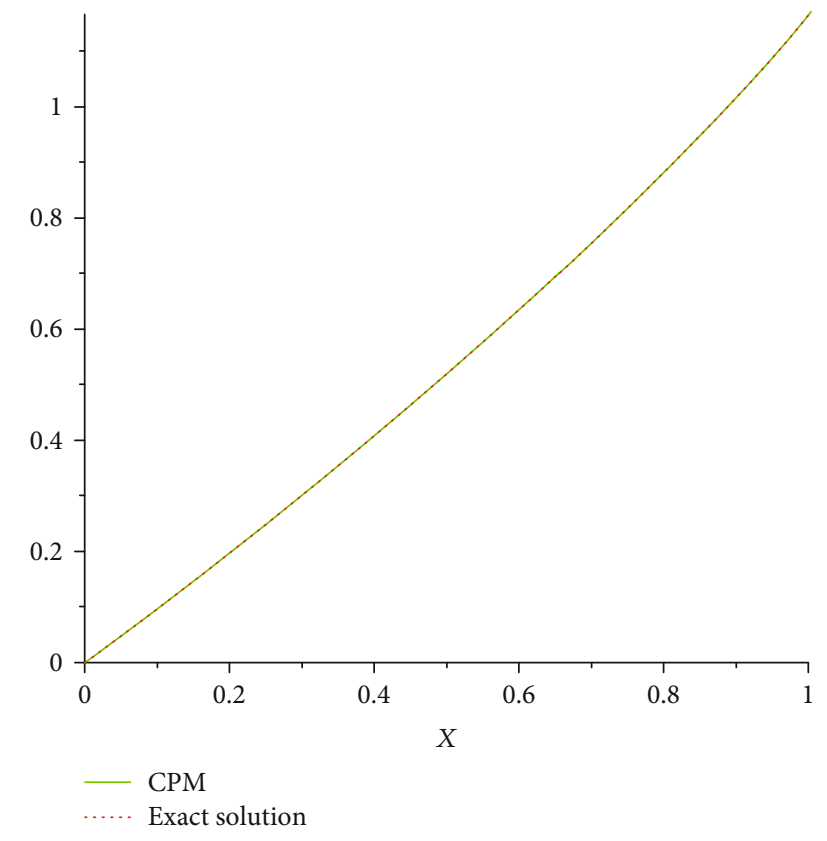

FIGURE 1: The solution graph of example 1. (a) Exact solution and (b) CPM solution.

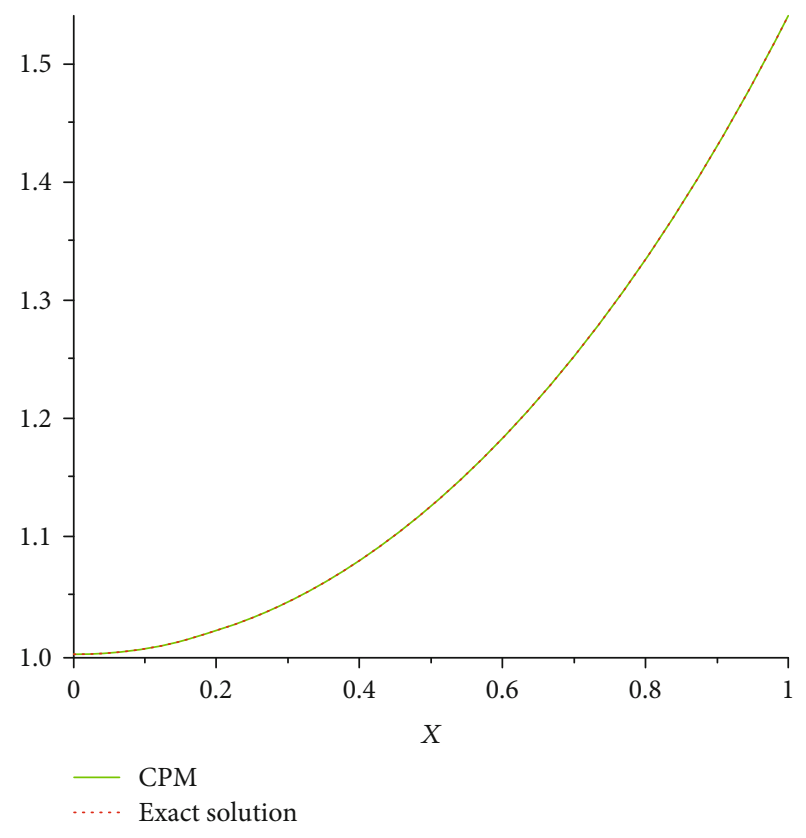

FIgURE 2: The solution graph of example 1. (a) Exact solution and (b) CPM solution. 


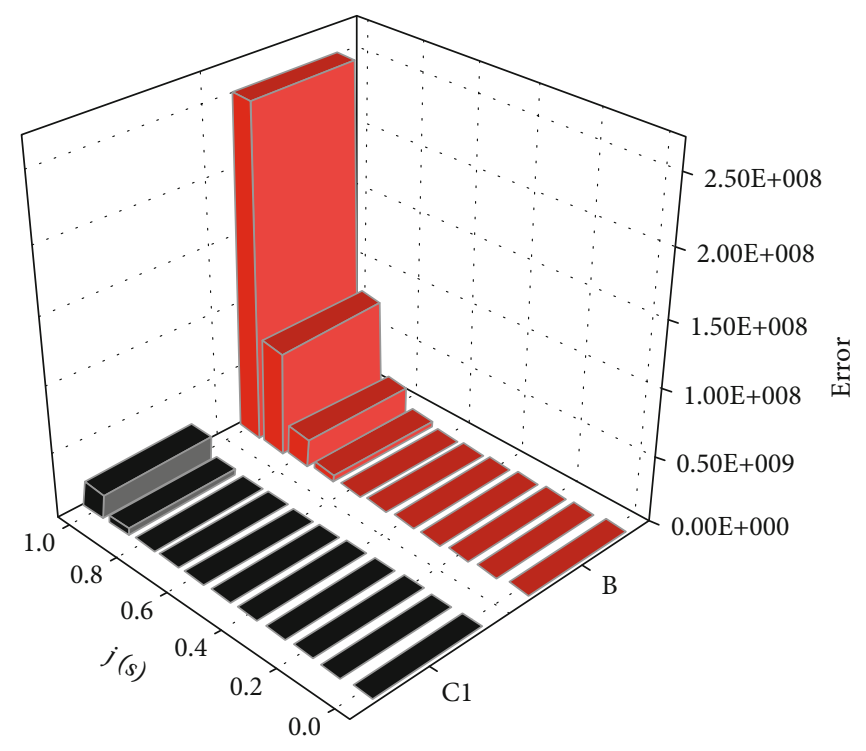

CPM

OTM

Figure 3: CPM and OTM error graph of Section 4.1.

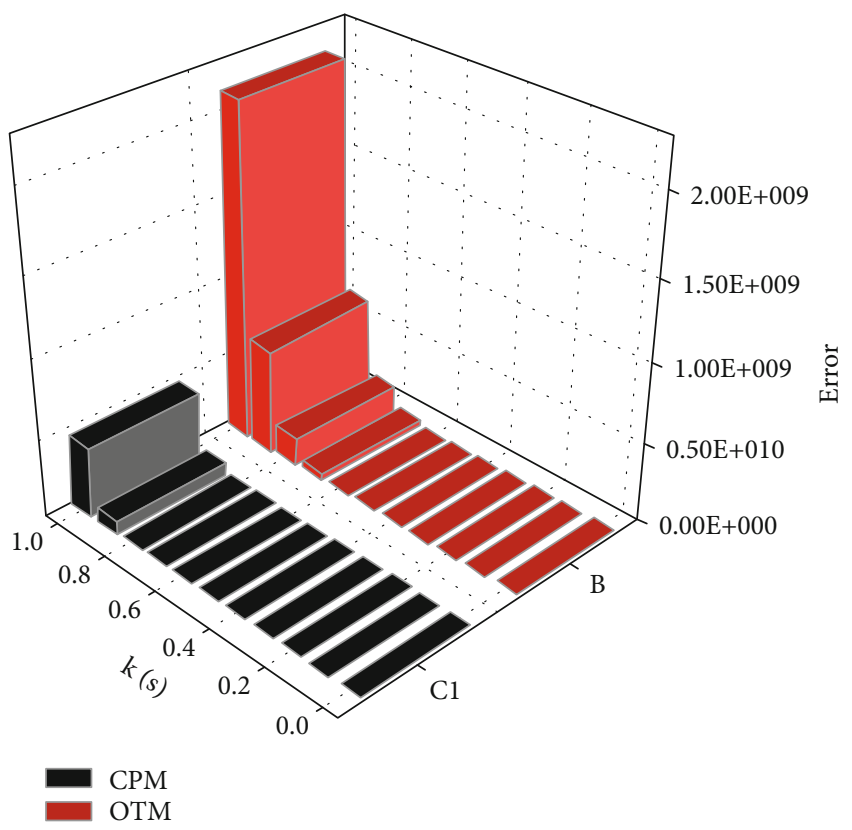

Figure 4: CPM and OTM error graph of Section 4.1.

A function $j(s) \in L_{2}[0,1]$, in terms of Chebyshev shifted polynomials described as

$$
j(s)=\sum_{n=1}^{\infty} c_{n} \widehat{R}_{n}(s)
$$

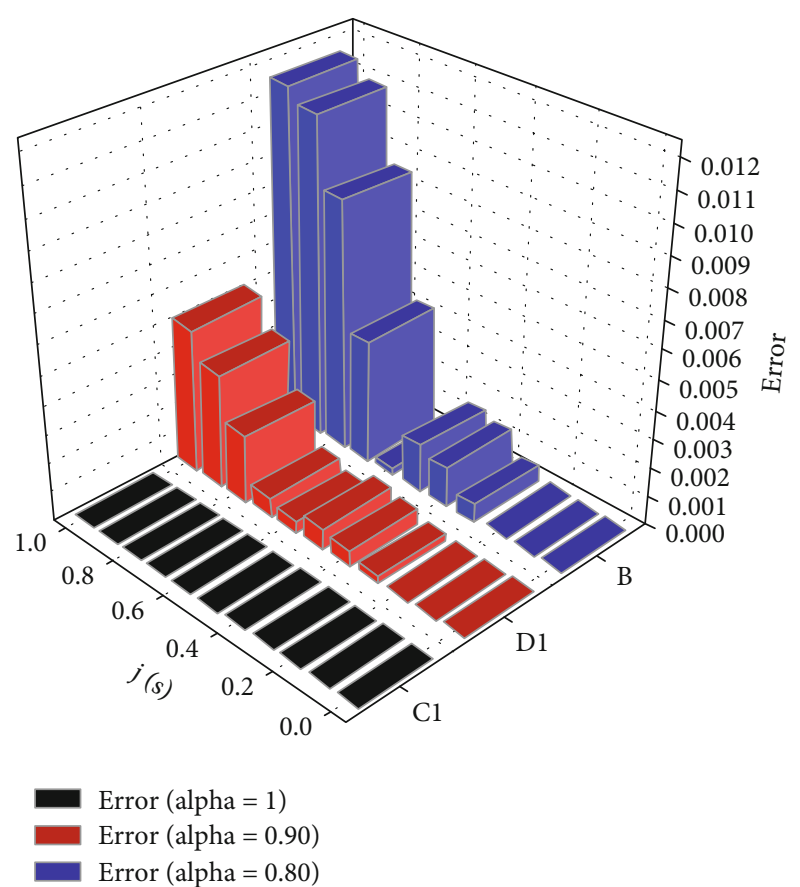

Figure 5: The absolute error graph of Section 4.1 at a different fractional order.

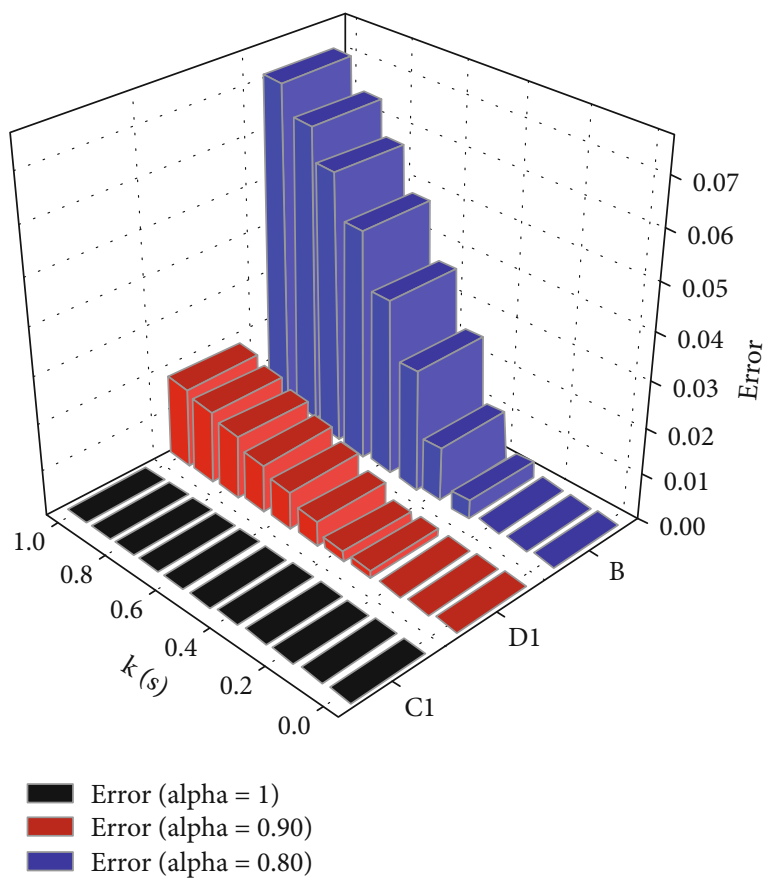

Figure 6: The absolute error graph of Section 4.1 at a different fractional order. 
TABLE 3: Exact and CPM solutions of Section 4.2 at $m=3$.

\begin{tabular}{lcccc}
\hline$s$ & Exact $j(s)$ & Exact $k(s)$ & CPM solution $j(s)$ & CPM solution $k(s)$ \\
\hline 0.05 & 0.047500000000000 & -0.002375000000000 & 0.047500000050000 & -0.002375000000000 \\
0.15 & 0.127500000000000 & -0.019125000000000 & 0.127500000100000 & -0.019124999970000 \\
0.25 & 0.187500000000000 & -0.046875000000000 & 0.187500000100000 & -0.046874999940000 \\
0.35 & 0.227500000000000 & -0.079625000000000 & 0.227500000100000 & -0.079624999950000 \\
0.45 & 0.247500000000000 & -0.111375000000000 & 0.247500000000000 & -0.111374999900000 \\
0.55 & 0.247500000000000 & -0.136125000000000 & 0.247500000000000 & -0.136125000000000 \\
0.65 & 0.227500000000000 & -0.147875000000000 & 0.227500000000000 & -0.147875000000000 \\
0.75 & 0.187500000000000 & -0.140625000000000 & 0.187500000000000 & -0.140625000000000 \\
0.85 & 0.127500000000000 & -0.108375000000000 & 0.127500000000000 & -0.108375000000000 \\
0.95 & 0.047500000000000 & -0.045125000000000 & 0.047499999999999 & -0.045125000100000 \\
\hline
\end{tabular}

TABLE 4: Error comparison of CPM versus other methods of Section 4.2.

\begin{tabular}{lcccc}
\hline$s$ & Error $\left(j_{\mathrm{CPM}}\right)$ & Error $\left(k_{\mathrm{CPM}}\right)$ & Error $\left(j_{\mathrm{SCM}}\right)$ & Error $\left(k_{\mathrm{SCM}}\right)$ \\
\hline 0.05 & $5.0000000000 E-11$ & $0.0000000000 E+00$ & $8.17886 E-8$ & $4.14502 E-8$ \\
0.15 & $1.0000000000 E-10$ & $3.0000000000 E-11$ & $3.00945 E-9$ & $5.20012 E-8$ \\
0.25 & $1.0000000000 E-10$ & $6.0000000000 E-11$ & $5.66834 E-8$ & $1.53079 E-7$ \\
0.35 & $1.0000000000 E-10$ & $5.0000000000 E-11$ & $3.81977 E-8$ & $1.82626 E-7$ \\
0.45 & $0.0000000000 E+00$ & $1.0000000000 E-10$ & $3.16220 E-8$ & $6.42170 E-7$ \\
0.55 & $0.0000000000 E+00$ & $0.0000000000 E+00$ & $6.05974 E-8$ & $6.19236 E-7$ \\
0.65 & $0.0000000000 E+00$ & $0.0000000000 E+00$ & $9.63834 E-9$ & $1.37882 E-7$ \\
0.75 & $0.0000000000 E+00$ & $0.0000000000 E+00$ & $4.55344 E-8$ & $1.53242 E-7$ \\
0.85 & $0.0000000000 E+00$ & $0.0000000000 E+00$ & $8.32363 E-8$ & $1.18939 E-8$ \\
0.95 & $5.0000000000 E-11$ & $1.0000000000 E-10$ & $9.10621 E-8$ \\
\hline
\end{tabular}

The Chebyshev shifted polynomials first $(m+1)$ terms are considered as

$$
\begin{gathered}
j_{m}(s)=\sum_{n=0}^{m} c_{n} \widehat{R}_{n}(s) \\
\frac{d^{\alpha}}{d s^{\alpha}}\left(\sum_{n=0}^{m} c_{n} \widehat{R}_{n}(s)\right)+\sum_{n=0}^{m} c_{n} \widehat{R}_{n}(s)+\int_{u_{0}}^{s}\left(\sum_{n=0}^{m} c_{n} \widehat{R}_{n}(s)\right) d s=g(s, j) .
\end{gathered}
$$

For finding the system of equations, we have

$\frac{d^{\alpha}}{d s^{\alpha}}\left(\sum_{n=0}^{m} c_{n} \widehat{R}_{n}\left(s_{i}\right)\right)+\sum_{n=0}^{m} c_{n} \widehat{R}_{n}\left(s_{i}\right)+\int_{s_{0}}^{s}\left(\sum_{n=0}^{m} c_{n} \widehat{R}_{n}\left(s_{i}\right)\right) d u=g\left(s_{i}, j\right)$.

whereas

$$
s_{i}=\frac{i-0.5}{2^{k-1} M}
$$

I solved the resultant system using maple software, which provide CPM solution for the given problem.

\section{Numerical Representation}

4.1. Problem. Consider the nonlinear FIDE system having B.Cs $j(0)=0, k(0)=1$

$$
\begin{array}{r}
D^{\alpha} j(s)+\frac{1}{2}\left(\frac{d k}{d s}\right)^{2}-\int_{0}^{s}[(s-t) k(t)+k(t) j(t)] d t=1, \\
D^{\alpha} k(s)+s j(s)-\int_{0}^{s}\left[(s-t) j(t)+k^{2}(t)\right] d t=2 s,
\end{array}
$$

having $j(s)=\sinh (s), k(s)=\cosh (s)$ as the exact solution at $\alpha=1$.

The exact solution and numerical results obtained by means of the proposed method are shown in Table 1. The absolute error comparison of our method and those obtained from OTM is given in Table 2. The behavior of the exact solution and approximate solution (our method) of this example when $\alpha=1$ is presented in Figures 1 and 2 whereas the error comparison of CPM and OTM can be observed in Figures 3 and 4. The graphical representation for different fractional order of $\alpha$ is seen in Figures 5 and 6 which confirm that the solution converge to the exact solution as the value of $\alpha$ converges from the fractional order to the integer order. 


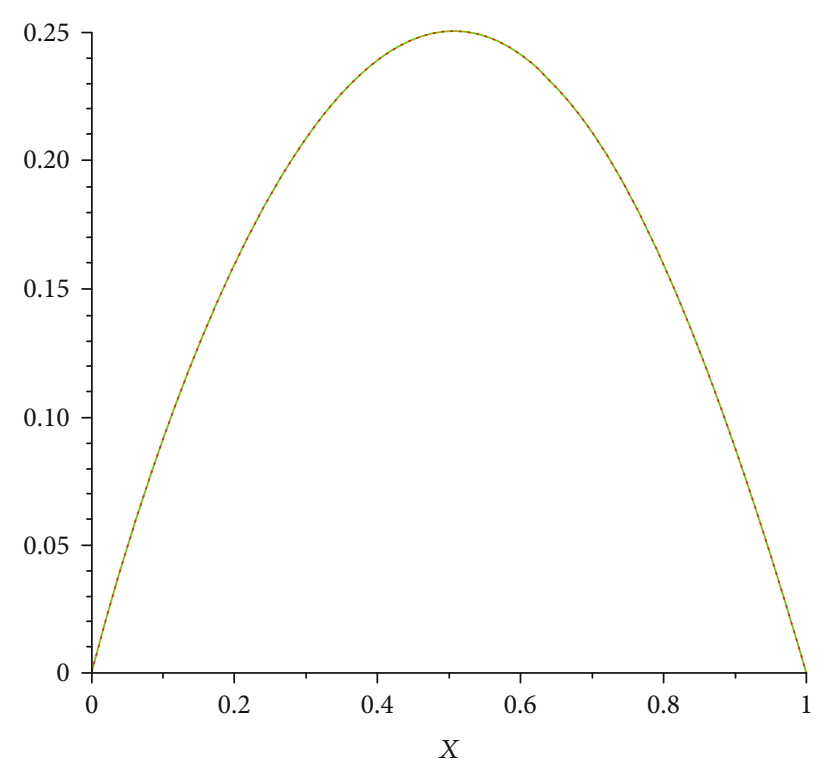

CPM

Exact solution

FIGURE 7: The solution graph of example 2. (a) Exact solution and (b) CPM solution.

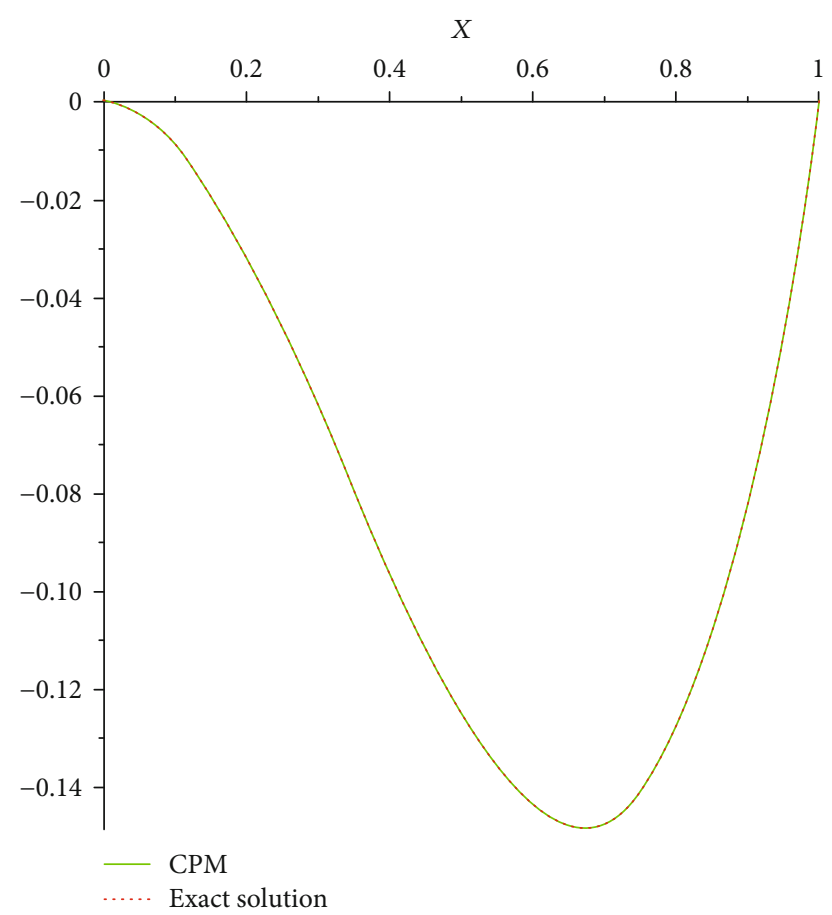

Figure 8: The solution graph of example 2. (a) Exact solution and (b) CPM solution.

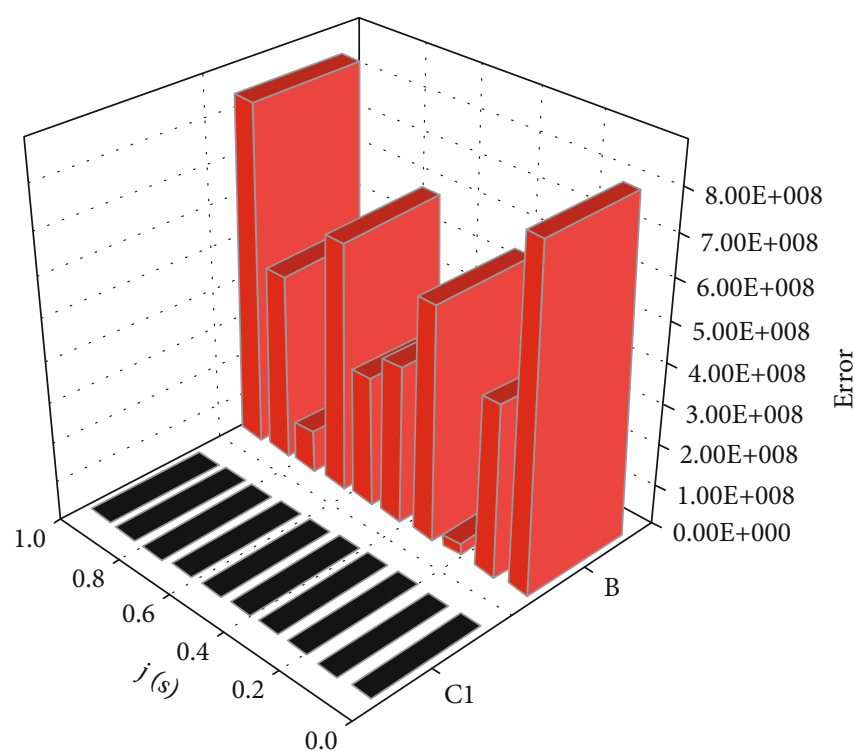

CPM

SCM

Figure 9: CPM and SCM error graph of Section 4.2.

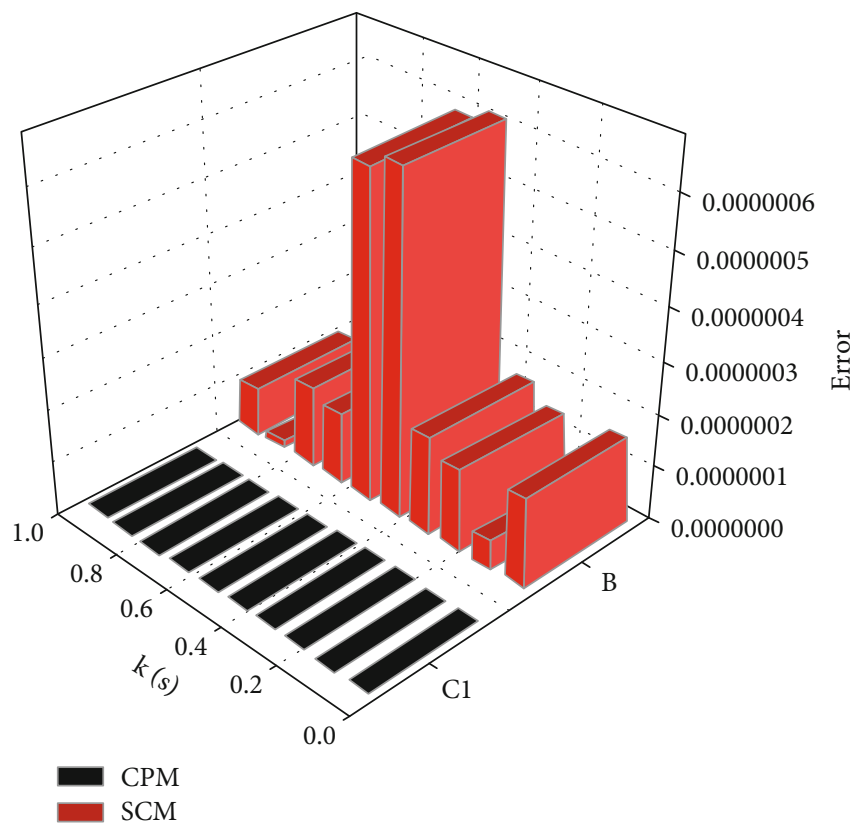

FIGURE 10: CPM and SCM error graph of Section 4.2.

4.2. Problem. Consider the FIDE system with B.Cs $j(0)=$ $j(1)=0, k(0)=k(0)=0$

$$
\begin{array}{r}
D^{\alpha} j(s)+k^{2}(s)+\frac{s}{2} \frac{d k}{d s}-\int_{0}^{s}((s-t) k(t)+j(t) k(t)) d t=g_{2}(s), \\
D^{\alpha} k(s)+j^{2}(s)-\int_{0}^{s}\left((s-t) j(t)-k^{2}(t)+j^{2}(t)\right) d t=g_{1}(s),
\end{array}
$$


TABle 5: Exact versus CPM solution of Section 4.3 at $m=10$.

\begin{tabular}{lcccc}
\hline$s$ & Exact $j(s)$ & Exact $k(s)$ & CPM solution $j(s)$ & CPM solution $k(s)$ \\
\hline 0 & 1.0000000000 & -1.0000000000 & 1.0000000000 & -1.0000000000 \\
0.1 & 1.2051709180 & -1.0051709180 & 1.2051709180 & -1.0051709180 \\
0.2 & 1.4214027580 & -1.0214027580 & 1.4214027580 & -1.0214027580 \\
0.3 & 1.6498588080 & -1.0498588075 & 1.6498588080 & -1.0498588075 \\
0.4 & 1.8918246980 & -1.0918246976 & 1.8918246980 & -1.0918246976 \\
0.5 & 2.1487212710 & -1.1487212707 & 2.1487212710 & -1.1487212707 \\
0.6 & 2.4221188000 & -1.2221188003 & 2.4221188000 & -1.2221188003 \\
0.7 & 2.7137527070 & -1.3137527074 & 2.7137527070 & -1.3137527074 \\
0.8 & 3.0255409280 & -1.4255409284 & 3.0255409280 & -1.4255409284 \\
0.9 & 3.3596031110 & -1.5596031111 & 3.3596031111 & -1.5596031109 \\
1.0 & 3.7182818280 & -1.7182818284 & 3.7182818284 & -1.7182818275 \\
\hline
\end{tabular}

TABLE 6: Error comparison of CPM versus other methods of Section 4.3 at $m=10$.

\begin{tabular}{lcccc}
\hline$s$ & Error $\left(j_{\mathrm{CPM}}\right)$ & Error $\left(k_{\mathrm{CPM}}\right)$ & Error $\left(j_{\text {OTM }}\right)$ & Error $\left(k_{\text {OTM }}\right)$ \\
\hline 0 & $0.0000000000 E+00$ & $0.0000000000 E+00$ & $0.00 E+00$ & $0.00 E+00$ \\
0.1 & $8.1999412724 E-17$ & $8.2624090006 E-17$ & $2.22 E-16$ & $0.00 E+00$ \\
0.2 & $3.8043301351 E-15$ & $3.8490016915 E-15$ & $4.44 E-16$ & $6.66 E-16$ \\
0.3 & $1.9076326419 E-14$ & $1.9470658817 E-14$ & $4.55 E-14$ & $4.55 E-14$ \\
0.4 & $3.8309289099 E-14$ & $3.9767165526 E-14$ & $1.09 E-12$ & $1.09 E-12$ \\
0.5 & $5.6578756405 E-14$ & $6.0124928716 E-14$ & $1.28 E-11$ & $1.28 E-11$ \\
0.6 & $3.2264909603 E-13$ & $3.2822178282 E-13$ & $9.57 E-11$ & $9.57 E-11$ \\
0.7 & $6.8521655232 E-12$ & $6.8380840814 E-12$ & $5.26 E-10$ & $5.26 E-10$ \\
0.8 & $7.2715580869 E-11$ & $7.2515818424 E-11$ & $2.30 E-9$ & $2.30 E-9$ \\
0.9 & $4.8186768333 E-10$ & $4.8069973992 E-10$ & $8.49 E-9$ & $8.49 E-9$ \\
1.0 & $2.3551879379 E-09$ & $2.3502526547 E-9$ & $2.73 E-8$ & $2.73 E-8$ \\
\hline
\end{tabular}

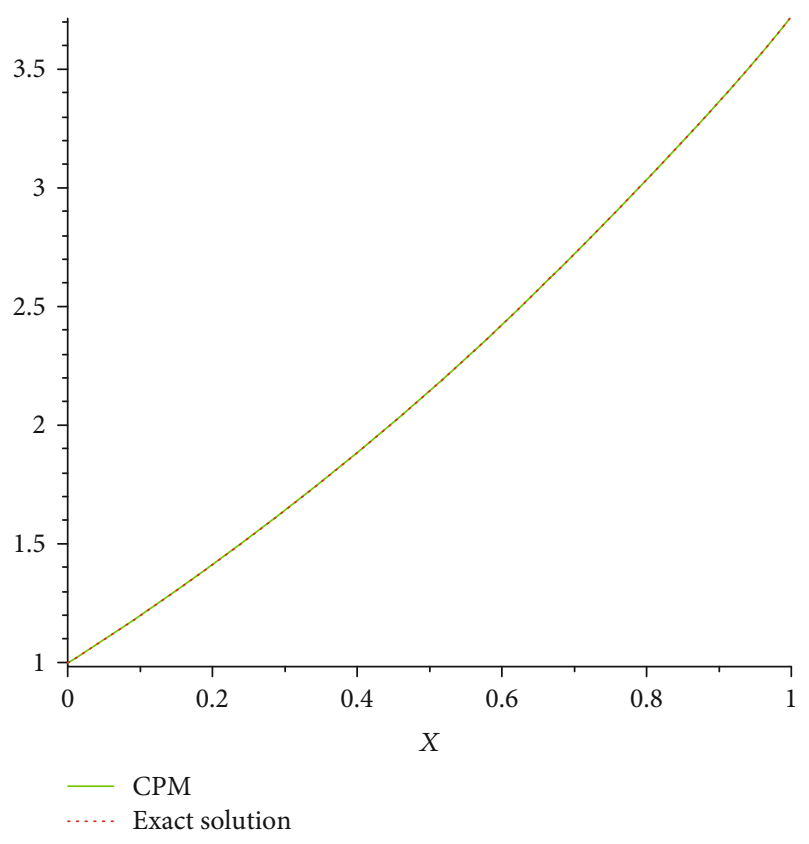

FIGURE 11: The solution graph of example 3. (a) Exact solution and (b) CPM solution.

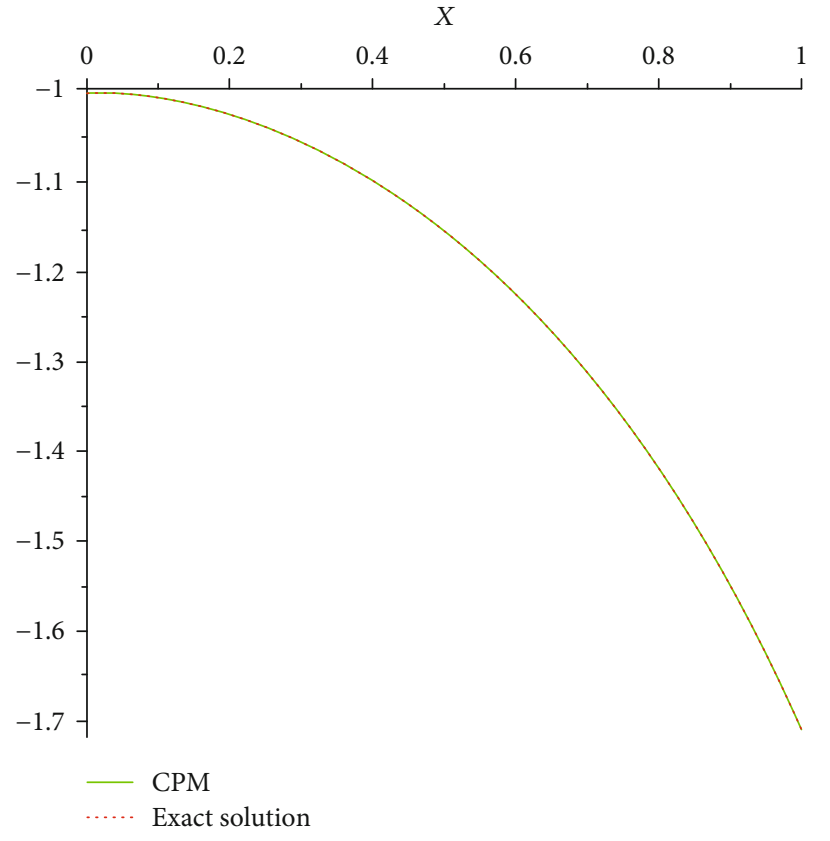

FIGURE 12: The solution graph of example 3. (a) Exact solution and (b) CPM solution. 


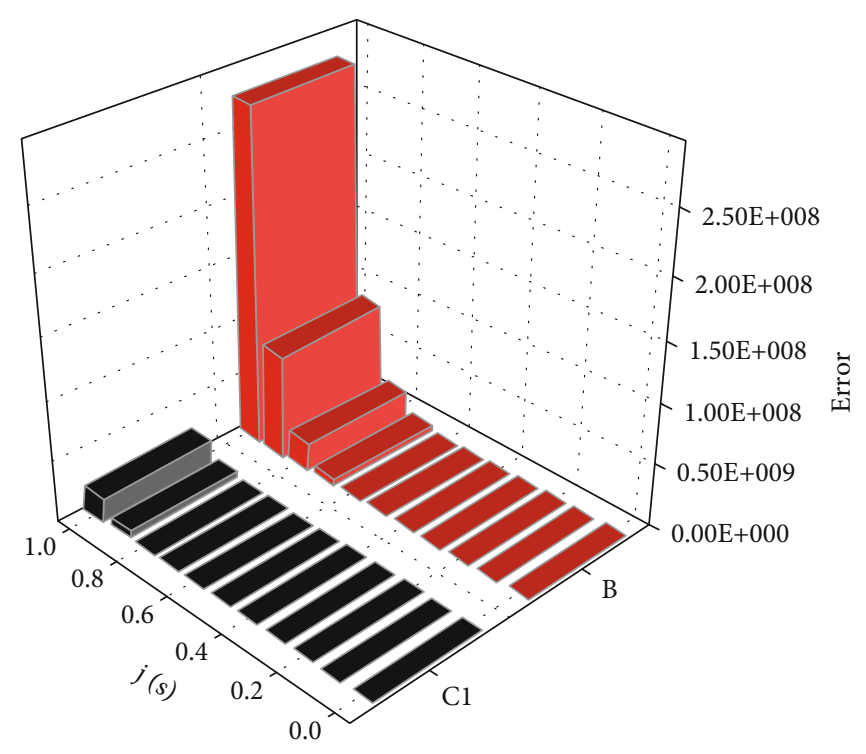

- $\mathrm{CPM}$

OTM

Figure 13: CPM and OTM error graph of Section 4.3.

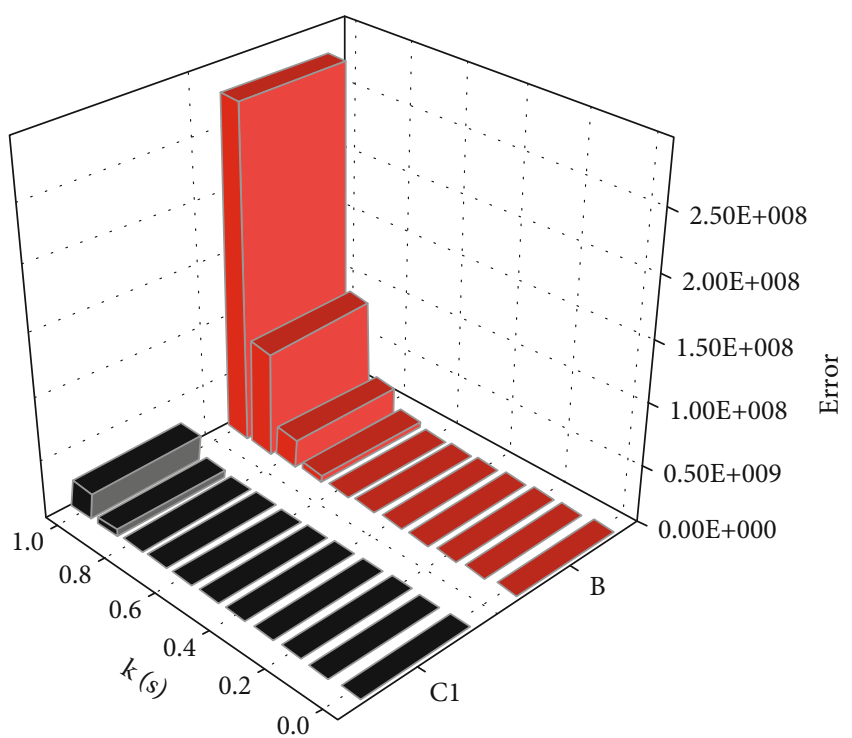

CPM

OTM

FIGURE 14: CPM and OTM error graph of Section 4.3.

with the exact solution $\left(j(s)=s-s^{2}, k(s)=s^{3}-s^{2}\right)$ at $\alpha=2$, where

$$
\begin{aligned}
& g_{1}(s)=\frac{7}{6} s^{6}-\frac{49}{20} s^{5}+\frac{4}{3} s^{4}+\frac{3}{2} s^{3}-s^{2}-2, \\
& g_{2}(s)=\frac{s^{7}}{7}-\frac{s^{6}}{3}+\frac{19}{12} s^{4}-\frac{5}{2} s^{3}+s^{2}+6 s-2 .
\end{aligned}
$$

In Table 3, we give the numerical values of the exact solution and CPM solution for $m=3$. The absolute errors

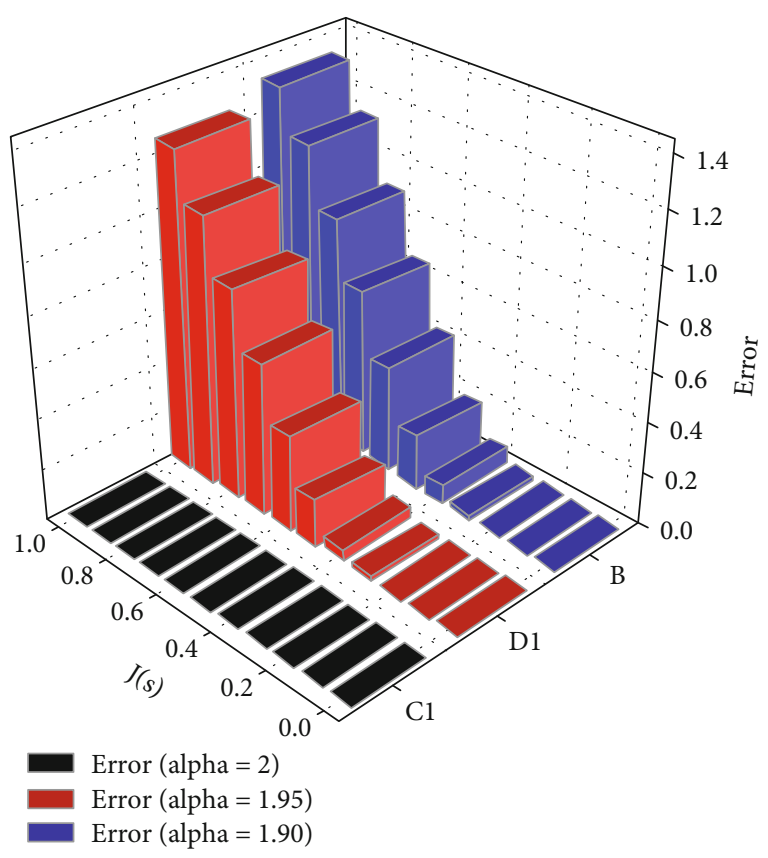

FIgURE 15: The absolute error graph of Section 4.3 at a different fractional order.

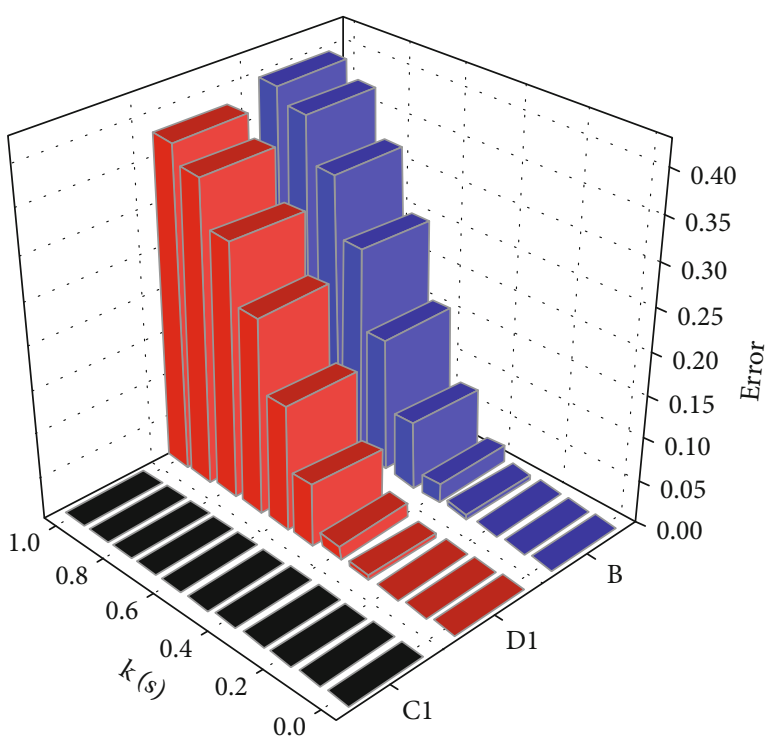

Error $($ alpha $=2)$
Error $($ alpha $=1.95)$
Error $($ alpha $=1.90)$

FIgURE 16: The absolute error graph of Section 4.3 at a different fractional order.

obtained by the present method are compared with SCM in Table 4. We compare the actual and estimated solution in Figures 7 and 8 which tells us that both the solutions are quite close to each other. Also, Figures 9 and 10 display the error comparison of CPM and SCM which verify that our method is in good agreement with the exact solution. 
4.3. Problem. Consider the nonlinear FIDE system having B.Cs $j(0)=1, j^{\prime}(0)=2, k(0)=-1$, and $k^{\prime}(0)=0$

$$
\begin{aligned}
D^{\alpha} j(s)+\frac{1}{2}\left(\frac{d k}{d s}\right)^{2}-\frac{1}{2} \int_{0}^{s}\left[j^{2}(t)+k^{2}(t)\right] d t & =1-\frac{1}{3} s^{3}, \\
D^{\alpha} k(s)+s j(s)-\frac{1}{4} \int_{0}^{s}\left[j^{2}(t)-k^{2}(t)\right] d t & =s^{2}-1,
\end{aligned}
$$

having $j(s)=s+e^{s}, k(s)=s-e^{s}$ as the exact solution.

To solve this example, we implement the method suggested in Section 4 for $\alpha=2$ with $m=10$. The exact solution and estimated solution by CPM are presented in Table 5 . The absolute error of our method and those obtained from OTM are given in Table 6. In Figures 11 and 12, it is clear that the numerical solution of the proposed method is in good contact with the exact solution. In order to illustrate the effectiveness of CPM, the error comparison with OTM is shown in Figures 13 and 14. Also, in Figures 15 and 16, we can obtain that as $\alpha \longrightarrow 2$ the estimated solution approach to the exact solutions.

\section{Conclusion}

In this work, we implemented the Chebyshev pseudospectral method for solving nonlinear fractional integral and integrodifferential equation systems. The proposed technique reduces this type of systems to the solution of the system of linear and nonlinear algebraic equations. Special attention is given to study the convergence of the proposed method. The results that we get by implementing the suggested technique are in excellent agreement with the exact solution and show more accuracy than the solution obtained using other methods. Also, from the presented figures, it is easy to conclude that the CPM error converges quickly as compared to other methods. The computation work in this article is done using Maple.

\section{Data Availability}

The numerical data used to support the findings of this study are included within the article.

\section{Conflicts of Interest}

The authors declare that they have no competing interests.

\section{Authors' Contributions}

All authors jointly worked on the results, and they read and approved the final manuscript.

\section{Acknowledgments}

This study is supported by the Central Department of Mathematics, Tribhuvan University, Kirtipur, Kathmandu, Nepal.

\section{References}

[1] A. Loverro, Fractional calculus: history, definitions and applications for the engineer, Rapport technique, Univeristy of Notre Dame: Department of Aerospace and Mechanical Engineering, 2004.

[2] R. L. Bagley and P. J. Torvik, "Fractional calculus in the transient analysis of viscoelastically damped structures," AIAA Journal, vol. 23, no. 6, pp. 918-925, 1985.

[3] R. T. Baillie, T. Bollerslev, and H. O. Mikkelsen, "Fractionally integrated generalized autoregressive conditional heteroskedasticity," Journal of Econometrics, vol. 74, no. 1, pp. 3-30, 1996.

[4] F. Mainardi, "Fractional calculus: some basic problems in continuum and statistical mechanics," 2012, https://arxiv.org/abs/ 1201.0863.

[5] T. S. Chow, "Fractional dynamics of interfaces between softnanoparticles and rough substrates," Physics Letters A, vol. 342, no. 1-2, pp. 148-155, 2005.

[6] Y. A. Rossikhin and M. V. Shitikova, "Applications of fractional calculus to dynamic problems of linear and nonlinear hereditary mechanics of solids," Applied Mechanics Reviews, vol. 50, no. 1, pp. 15-67, 1997.

[7] C. Li, X. Dao, and P. Guo, "Fractional derivatives in complex planes," Nonlinear Analysis: Theory, Methods \& Applications, vol. 71, no. 5-6, pp. 1857-1869, 2009.

[8] E. Guariglia, "Fractional calculus, zeta functions and Shannon entropy," Open Mathematics, vol. 19, no. 1, pp. 87-100, 2021.

[9] P. Sunthrayuth, N. H. Aljahdaly, A. Ali, R. Shah, I. Mahariq, and A. M. Tchalla, "Ф-Haar Wavelet Operational Matrix Method for Fractional Relaxation-Oscillation Equations Containing Ф-Caputo Fractional Derivative," Journal of Function Spaces, 2021.

[10] E. Guariglia and S. Silvestrov, "Fractional-Wavelet Analysis of Positive definite Distributions and Wavelets on $\mathscr{D}^{\prime}(\mathbb{C})$," in Engineering Mathematics II, Springer Proceedings in Mathematics \& Statistics, pp. 337-353, Springer, Cham. Switzerland, 2016.

[11] Y. Chen, Y. Yan, and K. Zhang, "On the local fractional derivative," Journal of Mathematical Analysis and Applications, vol. 362, no. 1, pp. 17-33, 2010.

[12] N. Alessa, K. Tamilvanan, K. Loganathan, and K. K. Selvi, "Hyers-Ulam Stability of Functional Equation Deriving from Quadratic Mapping in Non-Archimedean -Normed Spaces," Journal of Function Spaces, vol. 2021, Article ID 9953214, 10 pages, 2021.

[13] M. Arfan, I. Mahariq, K. Shah, T. Abdeljawad, G. Laouini, and P. O. Mohammed, "Numerical computations and theoretical investigations of a dynamical system with fractional order derivative," Alexandria Engineering Journal, 2021.

[14] D. Kaur, P. Agarwal, M. Rakshit, and M. Chand, "Fractional calculus involving (p, q)-Mathieu type series," Applied Mathematics and Nonlinear Sciences, vol. 5, no. 2, pp. 15-34, 2020.

[15] W. H. Deng and C. P. Li, "Chaos synchronization of the fractional Lu system," Physica A: Statistical Mechanics and its Applications, vol. 353, pp. 61-72, 2005.

[16] K. B. Oldham, "The reformulation of an infinite sum via semiintegration," SIAM Journal on Mathematical Analysis, vol. 14, no. 5, pp. 974-981, 1983.

[17] T. T. Hartley, C. F. Lorenzo, and H. Killory Qammer, "Chaos in a fractional order Chua's system," IEEE Transactions on 
Circuits and Systems I: Fundamental Theory and Applications, vol. 42, no. 8, pp. 485-490, 1995.

[18] E. Baskin and A. Iomin, "Electro-chemical manifestation of nanoplasmonics in fractal media," Central European Journal of Physics, vol. 11, no. 6, pp. 676-684, 2013.

[19] Y. Z. Povstenko, "Thermoelasticity that uses fractional heat conduction equation," Journal of Mathematical Sciences, vol. 162, no. 2, pp. 296-305, 2009.

[20] A. H. Bhrawy and M. A. Zaky, "A method based on the Jacobi tau approximation for solving multi-term time- space fractional partial differential equations," Journal of Computational Physics, vol. 281, pp. 876-895, 2015.

[21] R. Amin, I. Mahariq, K. Shah, M. Awais, and F. Elsayed, "Numerical solution of the second order linear and nonlinear integro-differential equations using Haar wavelet method," Arab Journal of Basic and Applied Sciences, vol. 28, no. 1, pp. 11-19, 2021.

[22] V. V. Kulish and J. L. Lage, "Application of fractional calculus to fluid mechanics," Journal of Fluids Engineering, vol. 124, no. 3, pp. 803-806, 2002.

[23] I. B. Bapna and N. Mathur, "Application of fractional calculus in statistics," International Journal of Contemporary Mathematical Sciences, vol. 7, no. 17-20, pp. 849-856, 2012.

[24] A. Kurt, M. Şenol, O. Tasbozan, and M. Chand, "Two reliable methods for the solution of fractional coupled burgers' equation arising as a model of polydispersive sedimentation," Applied Mathematics and Nonlinear Sciences, vol. 4, no. 2, pp. 523-534, 2019.

[25] P. Agarwal, F. Qi, M. Chand, and G. Singh, "Some fractional differential equations involving generalized hypergeometric functions," Journal of Applied Analysis, vol. 25, no. 1, pp. 3744, 2019.

[26] E. Bonyah, A. Atangana, and M. Chand, "Analysis of 3D ISLM macroeconomic system model within the scope of fractional calculus," Chaos, Solitons \& Fractals: X, vol. 2, article 100007, 2019.

[27] M. Chand, P. Agarwal, and Z. Hammouch, "Certain sequences involving product of k-Bessel function," International Journal of Applied and Computational Mathematics, vol. 4, no. 4, pp. 1-9, 2018.

[28] L. Xu, J.-H. He, and Y. Liu, "Electrospun nanoporous spheres with Chinese drug," International Journal of Nonlinear Sciences and Numerical Simulation, vol. 8, no. 2, pp. 199-202, 2007.

[29] H. Wang, H. M. Fu, H. F. Zhang, and Z. Q. Hu, “A practical thermodynamic method to calculate the best glass-forming composition for bulk metallic glasses," International Journal of Nonlinear Sciences and Numerical Simulation, vol. 8, no. 2, pp. 171-178, 2007.

[30] T.-L. Bo, L. Xie, and X. J. Zheng, "Numerical approach to wind ripple in desert," International Journal of Nonlinear Sciences and Numerical Simulation, vol. 8, no. 2, pp. 223228, 2007.

[31] F. Z. Sun, M. Gao, S. H. Lei et al., "The fractal dimension of the fractal model of dropwise condensation and its experimental study," International Journal of Nonlinear Sciences and Numerical Simulation, vol. 8, no. 2, pp. 211-222, 2007.

[32] A. Akyuz-Dascioglu, "Chebyshev polynomial solutions of systems of linear integral equations," Applied Mathematics and Computation, vol. 151, no. 1, pp. 221-232, 2004.
[33] H. A. Zedan and E. Alaidarous, "Haar wavelet method for the system of integral equations," Abstract and Applied Analysis, vol. 2014, Article ID 418909, 9 pages, 2014.

[34] H. Almasieh and M. Roodaki, "Triangular functions method for the solution of Fredholm integral equations system," Ain Shams Engineering Journal, vol. 3, no. 4, pp. 411-416, 2012.

[35] N. Sahin, S. Yuzbasi, and M. Gulsu, “A collocation approach for solving systems of linear Volterra integral equations with variable coefficients," Computers \& Mathematics with Applications, vol. 62, no. 2, pp. 755-769, 2011.

[36] P. K. Sahu and S. S. Ray, "Legendre wavelets operational method for the numerical solutions of nonlinear Volterra integro-differential equations system," Applied Mathematics and Computation, vol. 256, pp. 715-723, 2015.

[37] K. Maleknejad, N. Aghazadeh, and M. Rabbani, "Numerical solution of second kind Fredholm integral equations system by using a Taylor-series expansion method," Applied Mathematics and Computation, vol. 175, no. 2, pp. 1229-1234, 2006.

[38] P. Roul and P. Meyer, "Numerical solutions of systems of nonlinear integro-differential equations by homotopy-perturbation method," Applied Mathematical Modelling, vol. 35, no. 9, pp. 4234-4242, 2011.

[39] S. Bushnaq, B. Maayah, S. Momani, and A. Alsaedi, "A reproducing kernel Hilbert space method for solving systems of fractional integrodifferential equations," Abstract and Applied Analysis, vol. 2014, Article ID 103016, 6 pages, 2014.

[40] J. Biazar, "Solution of systems of integral-differential equations by Adomian decomposition method," Applied Mathematics and Computation, vol. 168, no. 2, pp. 1232-1238, 2005.

[41] Y. Wang and L. Zhu, "Solving nonlinear Volterra integrodifferential equations of fractional order by using Euler wavelet method," Advances in Difference Equations, vol. 2017, no. 1, Article ID 27, 2017.

[42] J. Saberi-Nadjafi and M. Tamamgar, "The variational iteration method: a highly promising method for solving the system of integro-differential equations," Computers \& Mathematics with Applications, vol. 56, no. 2, pp. 346-351, 2008.

[43] X. Ma and C. Huang, "Spectral collocation method for linear fractional integro-differential equations," Applied Mathematical Modelling, vol. 38, no. 4, pp. 1434-1448, 2014.

[44] O. T, T. OA, A. JU, and O. ZO, "Numerical studies for solving fractional integro-differential equations by using least squares method and bernstein polynomials," Fluid Mechanics Open Access, vol. 3, no. 3, pp. 1-7, 2016.

[45] S. Abbasbandy, M. S. Hashemi, and I. Hashim, "On convergence of homotopy analysis method and its application to fractional integro-differential equations," Quaestiones Mathematicae, vol. 36, no. 1, pp. 93-105, 2013.

[46] A. Arikoglu and I. Ozkol, "Solutions of integral and integrodifferential equation systems by using differential transform method," Computers \& Mathematics with Applications, vol. 56, no. 9, pp. 2411-2417, 2008.

[47] M. Yi, L. Wang, and J. Huang, "Legendre wavelets method for the numerical solution of fractional integro- differential equations with weakly singular kernel," Applied Mathematical Modelling, vol. 40, no. 4, pp. 3422-3437, 2016.

[48] J.-H. He, Z.-B. Li, and Q.-1. Wang, "A new fractional derivative and its application to explanation of polar bear hairs," Journal of King Saud University-Science, vol. 28, no. 2, pp. 190-192, 2016. 